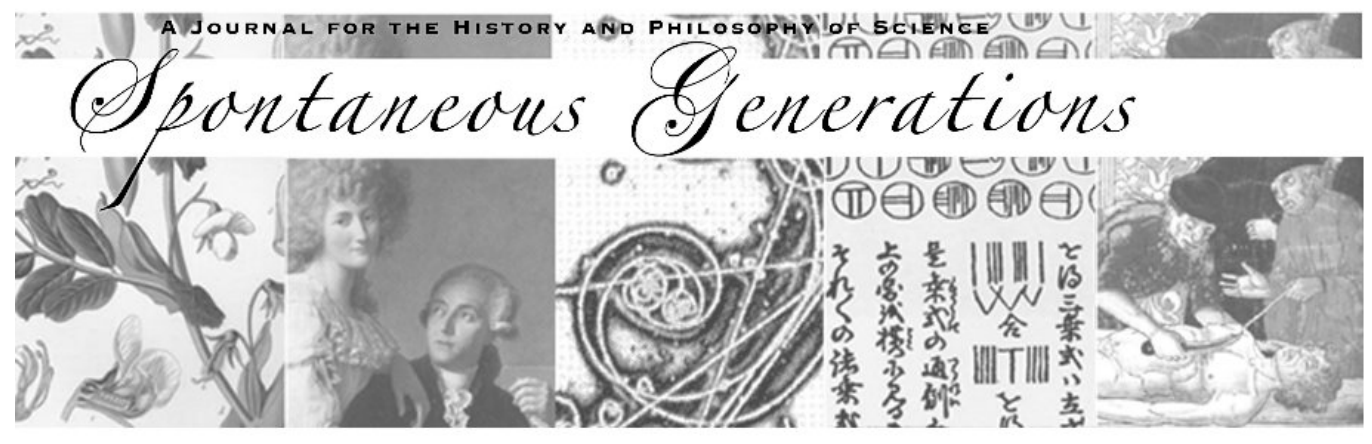

\title{
Material Culture and the Dobsonian Telescope
}

Author(s): Jessica Ellen Sewell and Andrew Johnston

Source: Spontaneous Generations: A Journal for the History and Philosophy of Science, Vol. 4, No. 1 (2010) 155-162.

Published by: The University of Toronto

DOI: $10.4245 /$ sponge.v4i1.11947

EDITORIAL OFFICES

Institute for the History and Philosophy of Science and Technology Room 316 Victoria College, 91 Charles Street West

Toronto, Ontario, Canada M5S 1K7

hapsat.society@utoronto.ca

Published online at jps.library.utoronto.ca/index.php/SpontaneousGenerations ISSN 19130465

Founded in 2006, Spontaneous Generations is an online academic journal published by graduate students at the Institute for the History and Philosophy of Science and Technology, University of Toronto. There is no subscription or membership fee. Spontaneous Generations provides immediate open access to its content on the principle that making research freely available to the public supports a greater global exchange of knowledge. 


\title{
Material Culture and the Dobsonian Telescope*
}

\author{
Jessica Ellen Sewell ${ }^{\dagger}$ and Andrew Johnston
}

This article examines the Dobsonian Telescope as an object of material culture, showing how starting with the materiality of a scientific instrument opens up new perspectives that are lost by focusing purely on its instrumentality. It argues that the simple design and homely materials of the Dobsonian telescope, as well as the gestures that it requires from its users, are at the core of its significance to the popularization of amateur astronomy and amateur telescope making.

Material culture is an interdisciplinary field of study that takes things seriously both as objects of study and as sources. From the beginnings of the field, material culture scholars have examined objects as repositories of culture, things in which the beliefs and practices of their makers are made visible (Prown 1982; Glassie 1999). Material culture scholars argue that objects function as cultural signs that can give us complex information about a culture that is often more truthful than those from traditional historical and anthropological sources because objects are created less self-consciously than texts or verbal statements. Objects are also more representative of a culture than texts, because objects are part of everyone's lives, while printed words reflect only the lives of the literate. However, objects are also more than signs, because they are functional and material as well as communicative. Recently, material culture scholars have tried to grapple with the stubborn materiality of things, the excess that remains above and beyond the immateriality of culture (Brown 2004; Graves-Brown 2000; Meskell 2005; Miller 2005). The aspect of this movement within material culture studies that is most

* Received 26 February 2010.

$\dagger$ Jessica Sewell is an Assistant Professor, specializing in material culture, in the American and New England Studies Program and the Department of Art History at Boston University. She is author of Women and the Everyday City: Public Space in San Francisco, 1890-1915 (Minneapolis: University of Minnesota Press, 2010).

$\ddagger$ Andrew Johnston is Associate Professor of Architecture at the Wentworth Institute of Technology. He is working on a book entitled Quicksilver Landscapes: Race, Space, and Power in the Mercury Mining Industry in California and has made two Dobsonian telescopes. 
pertinent to the study of scientific instruments is the call to explore the agency of objects, the way that objects are not merely things we act on, but also act upon us, helping to shape how we behave and who we are.

In this short essay, we explore how starting with the materiality of a scientific instrument leads us to ask interesting questions-questions which do not start with the instrumentality of a scientific instrument (how it functions in the service of science) but rather with its physicality. We are using the example of the Dobsonian telescope, a revolutionary instrument that helped to broaden the reach of amateur astronomy, reshape the nature of amateur observing, and encourage amateur astronomers to make their own telescopes. The Dobsonian changed the nature of amateur astronomers' investment in the practice of astronomy as well as in their telescopes. After introducing this instrument, we will explore two material questions engaged by it-the meanings and effects of the materials the Dobsonian mount is made of and the ways the viewer interacts physically with the telescope in the act of observing the heavens.

The Dobsonian telescope is a Newtonian reflector mounted on an alt-azimuth mount made of inexpensive materials. It was designed by John Dobson, a chemist turned Vedanta monk turned telescope maker and sidewalk astronomer, who started building telescopes in 1956. Over the following two decades, he combined numerous telescope-making insights into the telescope known as the Dobsonian, which became popular beginning in the late 1970s and early 1980s (Dickenson and Dyer 2008, 32-33; Kriege and Berry 1998, 7-28; for more information on Dobson, see Kriege and Berry 1998; Moser 1989; San Francisco Sidewalk Astronomers 2004a; and Sullivant 2001). In the Newtonian telescope, invented by Isaac Newton in 1668, light enters the tube of the scope, where it hits a large primary mirror that focuses it onto a smaller diagonal secondary mirror that reflects it to an eyepiece. Unlike the lenses of achromatic refracting telescopes, which distort colors, the mirrors of a Newtonian telescope reflect light without chromatic aberration. This quality, inherent in the design of a Newtonian telescope, allows the tube to be much shorter than a standard refractor of the same aperture, as a long focal length helps to minimize the chromatic aberration of a refractor. The short focal length possible in Newtonians allows telescopes to be of large aperture and a reasonable length, an important element in the design of the Dobsonian. The Dobsonian telescope uses an alt-azimuth mount, in which the telescope is able to move in two perpendicular axes, one vertical (altitude) and one horizontal (azimuth). This type of mount is mechanically less complex than an equatorial mount, in which one rotational axis is parallel to Earth's axis of rotation, allowing it to be driven with a simple clockwork motor to track celestial objects. 


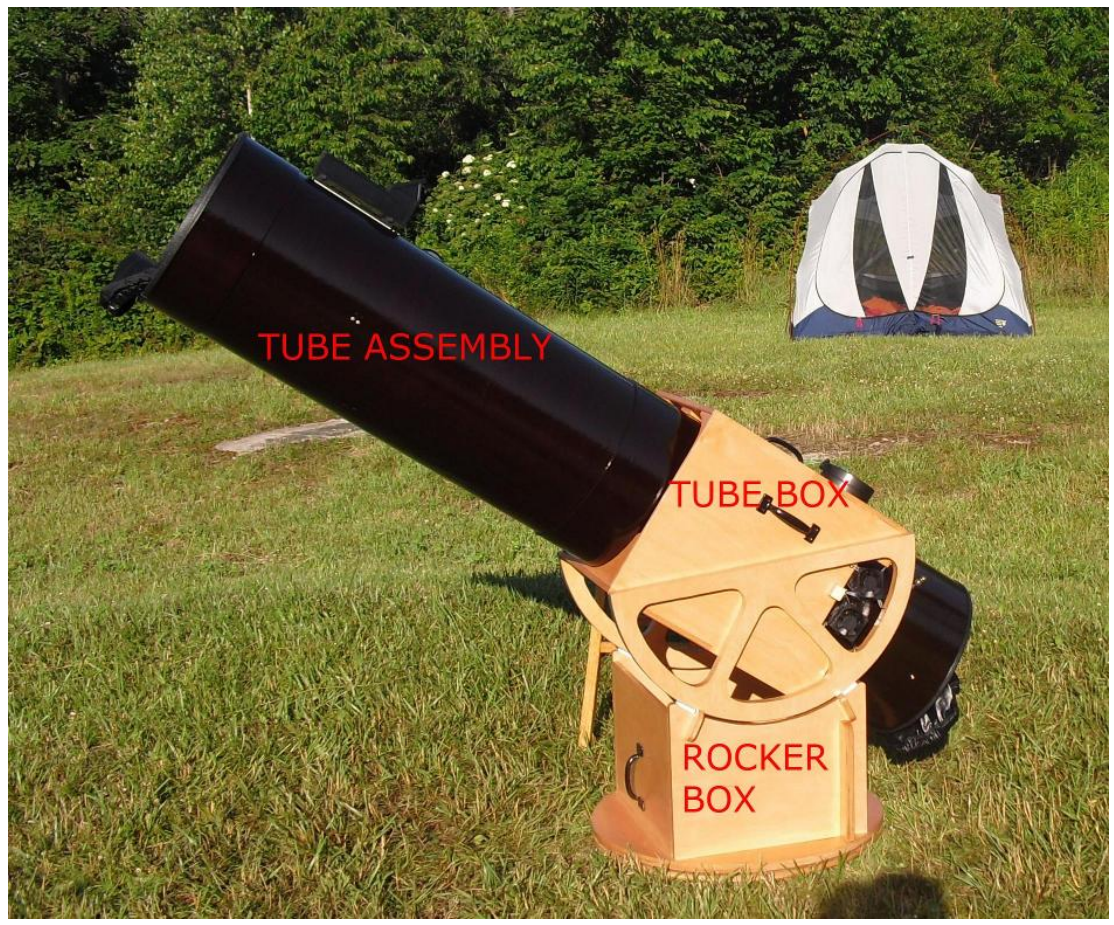

Figure 1. 12.5 Inch F4.3 Dobsonian Telescope made with sonotube, plywood, and hand-figured mirror by Andrew Johnston, 2009. Photo by authors.

The Dobsonian telescope consists of a tube assembly, usually made of a cardboard sonotube, inexpensive formwork for concrete columns, with a large parabolic mirror mounted at one end. This mirror focuses its image on a smaller diagonal mirror suspended in the center of the tube, which reflects the image to an eyepiece that sticks out of the side of the tube. The tube is usually held in a plywood box, the tube box, with circular or semi-circular rockers on two sides. These rockers sit on the rocker box, a plywood box with "cradle boards," curves cut to match the rockers. The rockers are faced on the bottom with Formica, which sits on small pieces of Teflon attached to the top of the cradle boards. Movement in the rocker provides the altitude element of the alt-azimuth mount. The rocker box sits on a ground board, attached by a center pivot, and rotates in relation to the ground board, once again on a combination of Formica and Teflon, to provide the azimuth element of the mount (see raycash.us/sfsidewalk/cdobplans.htm). The Formica and Teflon bearings allow the telescope to be easily moved in all directions because at low speeds the friction between these materials is low; as speed increases friction increases, keeping the movement steady; and when still these materials hold the telescope in place without slipping.

What is revolutionary about the Dobsonian telescope design is 
that it allows amateur astronomers to make large-aperture telescopes, colloquially known as light buckets, very cheaply. With large Dobsonian telescopes, amateur astronomers can easily view "faint fuzzies," the dim galaxies, nebulae, and star clusters that are difficult or impossible to view in telescopes of smaller aperture. The Dobsonian caused a revolution in the viewing habits of amateur astronomers, shifting observing from high-powered, detailed studies of the moon, planets, and double stars to low-powered, wide angle viewing of dim, "deep sky" objects for which the tracking made possible by equatorial mounts is less important (Dickenson and Dyer 2008, 32-33; Houston 1999; Kreige and Berry 1998, 18-19). Before the popularization of the Dobsonian, most amateur astronomers used German equatorial mounts for both refractor and reflector telescopes (Dickinson and Dyer 30-31; Kriege and Berry 1998, 19; Seronik 1998). Equatorial mounts are typically carefully machined objects, made of metal, and are therefore expensive. In comparison, the classic Dobsonian mount uses plywood, Formica, Teflon, and cardboard, what Dobson referred to as "just a pile of stuff" that is "held together by gravity and run on yogurt power" (Kreige and Berry 1997, 5). Similarly, while lenses for refracting telescopes must be made with optical glass of exacting qualities, Dobson showed that large, thin mirrors of sufficient quality for deep sky observing could be made and properly supported in the telescope, and that they could be made from inexpensive plate glass, available in large sizes from salvaged portholes and in smaller sizes from sources as mundane as the bottom of glass jugs. Dobson's own telescopes were made largely of scrap materials, including cardboard hose reels, cereal boxes, old portholes, and scrap lumber, although most of his followers use kits or new materials readily available at any home improvement store (Moser 1989; Kriege and Berry 1998; San Francisco Sidewalk Astronomers 2004b).

John Dobson's telescope design did not create a new way of seeing the heavens; what it did was to democratize the act of seeing. Arguably, the Dobsonian telescope has had a significant effect on the growth in the popularity of amateur astronomy; Kriege and Berry state that amateur astronomy grew fourfold between the 1970s and the 1980s, and the number of star parties, gatherings where amateur astronomers set up their telescopes to observe together, has increased dramatically since the late 1970s (Kriege and Berry 1998, 18). The inexpensiveness of the Dobsonian telescope, combined with the simplicity of building one, made amateur astronomy more accessible. In addition, the Dobsonian telescope made large telescopes with great light gathering ability significantly less expensive. Dobson himself prefers to call the Dobsonian telescope a "sidewalk telescope," and has spent nearly fifty years bringing telescopes to the masses on sidewalks, in national parks, and wherever he 
can convince someone to try looking through one (Moser 1989; San Francisco Sidewalk Astronomers 2004a; Sullivant 2001). The thriftiness and intuitive use of the Dobsonian telescope support his goal of making amateur astronomy accessible to all. In addition, by making larger telescopes inexpensive, the Dobsonian telescope has helped to make amateur astronomy more interesting and accessible, as home telescopes increasingly can focus in on the deep sky universe of nebulae and galaxies, rather than just planets and very bright objects.

Classic Dobsonian telescopes use inexpensive materials: a cardboard sonotube as the tube, and a mount made of plywood, Formica, and Teflon. The materials out of which the Dobsonian telescope is made are important for several reasons. They are inexpensive and easily worked without specialized tools, which is central to the low cost of Dobsonian telescopes. Unlike metal equatorial mounts, which typically require machining (and thus specialized skills and a specialized shop) a Dobsonian mount can be easily made with inexpensive hand tools. Because of the popularity of home improvement and amateur carpentry and the ubiquity of home power tools in American men's garages and workshops, the means to create a Dobsonian telescope are widespread (Gelber 1999). Dobsonians are also made of ordinary materials, easily acquired from home improvement stores or by scrounging scrap materials from building sites. While these practical aspects of the materials were undoubtedly central to Dobson's choice to use them, the cultural meanings of the materials are equally significant. Plywood, cardboard, and Formica are aggressively ordinary, even ugly, modern materials. Creating a precision scientific instrument from such pedestrian materials changes attitudes towards the instrument. A cardboard telescope is a less precious, less intimidating object than one made of more attractive and unusual materials.

A Dobsonian telescope is also an object that is often made by hand by the person who uses it for observing. The materials and means of making it help to encourage this, and in addition there were few commercial manufacturers making Dobsonians in the early years, so those who wanted a Dobsonian had to make it themselves. Even those who do buy commercial Dobsonians tend to customize them, making them unique. This process of making your own telescope has several consequences. It encourages the telescope maker to become part of a growing community of telescope makers, both in order to learn about the process of telescope making and to show off their finished product at festivals such as the Stellafane convention in Vermont (stellafane.org) and the Riverside Telescope Makers' Convention in California (www.rtmcastronomyexpo.org/general.html). The process of making a telescope also teaches the maker about how telescopes work. 
Making and mounting a mirror and properly aligning the primary mirror with the diagonal secondary mirror requires a rudimentary understanding of optics that is not required to use a commercial telescope. In addition, making the decisions about the focal length of the mirror and other aspects of the telescope's design requires a more sophisticated understanding of how different telescopes are suited to different types of observing. An amateur astronomer who has made his own Dobsonian not only knows more about telescopes than he did before making one, but he has also created a personal relationship with his own telescope that is deeper than one he would have with a commercial telescope. In the process of making the telescope, he has made it his in a very direct way. Not only does it reflect his handwork, but typically it also reflects the particular choices that make his telescope a little different than any other. The design of a Dobsonian telescope is in essence an open-source design, which is constantly being tweaked and refined by every person who makes one, and these refinements are shared throughout the community of telescope makers, through clubs, conventions, and online groups.

The mode of observing required by the Dobsonian mount reinforces the idea that Dobsonians are non-threatening, easy to use telescopes, worthy of their description in Astronomy as "the big, friendly dogs of observing that greet their masters with a visual tongue wash" (Harrington 2001). Typically, moving a telescope on an equatorial mount is a process of turning knobs. The telescope can only move in two directions, so moving it diagonally requires coordinating both knobs, like drawing on an etch-a-sketch. In contrast, one moves the Dobsonian telescope simply by pushing or pulling it lightly. In a well-made Dobsonian, the Formica and Teflon bearings are calibrated so that the telescope operator can gently nudge the telescope tube in a desired direction and it will move smoothly to any position in the sky and stay stationary when the force is removed. Larger Dobsonians are often essentially hugged by observers, who move the telescope with their body. The knobs and gears of an equatorial mount create a level of remove between the user and the telescope. The mount is a mechanical thing that an observer operates. In contrast, the Dobsonian telescope requires physical contact; it becomes, in the hands of experts, like an extension of their own bodies. The intimacy of viewing with a Dobsonian is in particularly marked contrast with current professional astronomy, which typically involves operating telescopes remotely. The experience of viewing with a Dobsonian adds a layer of intimacy and mastery to those already created by the experience of building it out of familiar items.

If we were to focus on the Dobsonian telescope as an instrument, we would ask what it allows a viewer to observe and how its movement affects that viewing. As an instrument, the Dobsonian's most important 


\title{
J. E. Sewell \& A. Johnston Material Culture \& the Dobsonian Telescope
}

qualities are that it is particularly well suited to the viewing of deep sky objects, somewhat imprecise for ideal viewing of planets and the moon, and that its mount tends to favor optical viewing rather than the use of astrophotography. As an instrument, it allowed amateur astronomers to discover what was possible when using a telescope that can gather a great deal of light. By examining the Dobsonian as a material object, we suggest that many of its most important qualities have little to do with its instrumentality. The Dobsonian telescope affects the physical, emotional, and social world of those who use and build one. It requires a new set of gestures for observing, gestures which engender a sense both of intimacy and of mastery in the user. To use a Dobsonian is to boss a telescope around, and almost to dance with it, rather than to operate it. Its materials further reinforce this emotional relationship of intimacy between astronomer and telescope. The Dobsonian is non-threatening and invites interaction because of the warmth and inexpensiveness of its materials. Using a Dobsonian, and especially making one, pulls many astronomers into a social world as well, an expanding universe of telescope making clubs, star parties, and online forums that epitomize the goals of sidewalk astronomy at the core of John Dobson's vision.

\author{
JESSICA ELLEN SEWELL \\ American and New England Studies Program \\ Boston University \\ 226 Bay State Rd. \\ Boston MA 02215 \\ jesewell@bu.edu \\ ANDREW JOHNSTON \\ Department of Architecture \\ Wentworth Institute of Technology \\ 550 Huntington Ave \\ Boston MA 02115 \\ johnstona@wit.edu
}

\section{REFERENCES}

Brown, Bill, ed. 2004. Things. Chicago: University of Chicago Press.

Brown, Sam. 1975. All About Telescopes. Tonawanda, NY: Edmunds Scientifics.

Dickinson, Terence, and Alan Dyer. 2008. The Backyard Astronomer's Guide. Richmond Hill, Ontario: Firefly Books.

Gelber, Steven M. 1999. Hobbies: Leisure and the Culture of Work in America. New York: Columbia University Press. 


\section{J. E. Sewell \& A. Johnston Material Culture \& the Dobsonian Telescope}

Glassie, Henry. 1999. Material Culture. Bloomington: Indiana University Press.

Graves-Brown, P.M., ed. 2000. Matter, Materiality and Modern Culture. London: Routledge.

Harrington, Phil. 2001. Who let the Dobs out? Dobsonian-style telescopes fetch deep-sky and planetary treats that welcome observers. Astronomy 20(9) (September): 74.

Houston, Walter Scott. 1999. Deep Sky Wonders. Cambridge, MA: Sky Publishing Corporation.

Kriege, David, and Richard Berry. 1998. The Dobsonian Telescope: A Practical Manual for Building Large Aperture Telescopes. Richmond, VA: Willmann-Bell.

Meskell, Lynn, ed. 2005. Archaeologies of Materiality. Malden: Blackwell.

Moser, Don. 1989. A Salesman for the heavens wants to rope you in. Smithsonian, 20(1) (April): 102-10.

Moser, Don. 2005. John Dobson: come one, come all. Share the sky with the father of astronomy. Smithsonian, 36(8) (November): 58-60.

Miller, Daniel, ed. 2005. Materiality. Durham: Duke University Press.

Prown, Jules David. 1982. Mind in Matter: An Introduction to Material Culture Theory and Method. Winterthur Portfolio, 17(1) (Spring): 1-19.

San Francisco Sidewalk Astronomers. 2004a. www.sfsidewalkastronomers.org.

San Francisco Sidewalk Astronomers. 2004b. John Dobson: A Brief Biography. www.sfsidewalkastronomers.org/index.php?page=john-dobson-abrief-biography.

San Francisco Sidewalk Astronomers and Ray Cash. n.d. Complete plans for building a Dobsonian telescope. raycash.us/sfsidewalk/cdobplans.htm.

Seronik, Gary. 1998. Teaching an old Dob new tricks. Sky \& Telescope 96(2) (August): 125-28.

Sullivant, Rosemary. 2001. On the road with John Dobson. For nearly 60 years, sharing the universe has been his mission. Astronomy 29(9) (September): 68. 